\title{
Seven-Nanometer Hexagonal Close Packed Cobalt Nanocrystals for High-Temperature Magnetic Applications through a Novel Annealing Process
}

\author{
C. Petit, ${ }^{\dagger}$ Z. L. Wang, ${ }^{\ddagger}$ and M. P. Pileni* ${ }^{\dagger} \dagger$ \\ Laboratoire des Matériaux Mésoscopiques et Nanométriques, UMR CNRS 7070, Université Pierre et Marie \\ Curie, 4 Place Jussieu, 75251 Paris Cedex, France, and School of Materials Science and Engineering, \\ Georgia Institute of Technology, Atlanta, Georgia 30332-0245
}

Received: May 12, 2005; In Final Form: June 10, 2005

\begin{abstract}
Seven-nanometer cobalt nanocrystals are synthesized by colloidal chemistry. Gentle annealing induces a direct structural transition from a low crystalline state to the hexagonal close packed (hcp) phase without changing the size, size distribution, and the lauric acid passivating layer. The hcp structured nanocrystals can be easily redispersed in solvent for further application and processing. We found that the magnetization at saturation and the magnetic anisotropy are strongly modified through the annealing process. Monolayer self-assembly of the hcp cobalt nanocrystals is obtained, and due to the dipolar interaction, ferromagnetic behavior close to room temperature has been observed. This work demonstrates a novel approach for obtaining small size hcp structured cobalt magnetic nanocrystals for many technological applications.
\end{abstract}

\section{Introduction}

The self-organization of ferromagnetic nanoparticles opens a new field of science and technology because they are potential candidates for magnetic data storage. Ideally in these materials, each well-separated nanocrystal is expected to correspond to one bit of information. ${ }^{1,2}$ However, thermal fluctuations in magnetization and dipolar magnetic interactions among nanocrystals in an array are important limitations for their use as a support for magnetic storage at room temperature. ${ }^{3}$ Actually, there is no clear solution to prevent the superparamagnetism phase transformation of nanocrystals of sizes below $10 \mathrm{~nm}$. For any synthesis route, further postsynthesis treatment is needed to obtain ferromagnetic nanoparticles stable at room temperature. Several attempts have been made to produce hard magnetic nanocrystals either dispersed in a solvent or self-organized into two-dimensional (2D) superlattices over the past few years. ${ }^{4-14}$ Each method has some advantages and disadvantages. Through the use of the organo-metallic route, it has been possible to make cobalt nanocrystals with high crystallinity. ${ }^{15}$ Their structure was either face centered cubic (fcc), a mixture of fcc-hcp, or the $\epsilon$ phase. ${ }^{9-11,15}$ To achieve a hard magnetic pure hcp structured Co for applications, the required annealing temperature is rather high, which inevitably induces changes in the self-organization and coalescing of the nanocrystals. This makes it difficult to compare the magnetic properties of an assembly of nanocrystals dispersed in a solvent with those of self-organized on a substrate. Soft chemistry using chemical reduction of cobalt ions with sodium borohydride is an easier route to synthesize cobalt nanocrystals. ${ }^{12-14}$ However, these nanoparticles usually display multiply twinned crystal structures and chemical contamination, like boron, from the reducing agent, which modifies their magnetic properties. As a consequence, analogous to the organometallic route, the magnetic anisotropy is too low for ferromagnetism at room temperature.

\footnotetext{
* Author to whom correspondence should be addressed. E-mail: pileni@ sri.jussieu.fr.

Université Pierre et Marie Curie.

$\doteqdot$ Georgia Institute of Technology.
}

In this article, we describe an approach that transforms the as-synthesized 7-nm cobalt nanocrystals, obtained by wet chemistry, directly from a low crystallinity state to the hcp state by keeping the size and coating agent on the surface. Fast and moderate annealing yields magnetic nanocrystals with a high magnetic anisotropy energy. The nanocrystals are dispersed in solution and used to form monolayers having a blocking temperature close to room temperature.

\section{Experimental Section}

2.1. Equipment. Transmission Electron Microscopy (TEM). A JEOL (100kV) model JEM 100CX II is used for the low resolution pictures and a Phillips CM20 with a CCD camera for the high-resolution TEM.

Magnetic Properties. The magnetic measurements are made using a commercial superconducing quantum interference device (SQUID) magnetometer (Cryogenic S600) from the SPEC (CEA-Saclay, France).

$X$-Ray Diffraction (XRD). XRD measurements were carried out using a STOE Stadi P goniometer with a Siemens Kristalloflex X-ray generator using a cobalt anode $(\lambda=1.7809 \AA)$. This apparatus is driven by a personal computer through a DACO-MP interface (Socabim, France).

2.2. Synthesis, Characterization, and Organization of the Cobalt Nanocrystals. The synthesis and characterization of cobalt nanocrystals by AOT reverse micelles have been extensively described in our previous papers. ${ }^{12-14}$ Cobalt bis(2-ethyl-hexyl)-sulfosuccinate, usually called $\mathrm{Co}(\mathrm{AOT})_{2}$, is solubilized in isooctane. For a water content, $w=\left[\mathrm{H}_{2} \mathrm{O}\right] /[\mathrm{AOT}]$, of 40 reverse micelles are formed. Through the use of overall concentrations of $10^{-1} \mathrm{M}$ for both $\mathrm{Co}(\mathrm{AOT})_{2}$ and $\mathrm{NaBH}_{4}$, cobalt nanocrystals are then produced through replacing water molecules by sodium tetrahydroboride. Immediately after borohydride addition, the micellar solution color turns from pink to black, indicating the formation of colloidal particles. It has been reported previously ${ }^{12}$ that the chemical scheme yielding the formation of the cobalt nanocrystals in mixed AOT/Co(AOT $)_{2}$ reverse micelles is similar to that reported in nonaqueous 
SCHEME 1: Soft Annealing of the Cobalt Nanocrystals

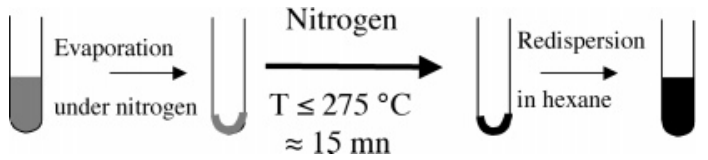

media. ${ }^{16,17}$ This has been explained by the fact that the nonaqueous solvent acts as a ligand, L, as follows

$$
\begin{gathered}
\mathrm{Co}^{2+}+2 \mathrm{NaBH}_{4}+n \mathrm{~L} \rightarrow(\mathrm{L})_{n} \mathrm{Co}\left(\mathrm{BH}_{4}\right)_{2}+2 \mathrm{Na}^{+} \\
(\mathrm{L})_{n} \mathrm{Co}\left(\mathrm{BH}_{4}\right)_{2} \rightarrow \mathrm{Co}+\mathrm{H}_{2}+\mathrm{B}_{2} \mathrm{H}_{6}+n \mathrm{~L}
\end{gathered}
$$

In fact, there is a clear analogy between the effect of the ligand and the effect of the functionalized surfactant on the chemical mechanism in reverse micelles. Addition of lauric acid, $\mathrm{C}_{12} \mathrm{H}_{25} \mathrm{COOH}$, to the micellar solution induces a covalent attachment with cobalt atoms located at the interface. The coated cobalt nanocrystals are then extracted from reverse micelles by ethanol addition, and the lauric-acid-coated particle precipitates. The particles are then washed and centrifuged several times with ethanol to remove all of the surfactant. Extended X-ray absorption fine structure (EXAFS) characterization ${ }^{13}$ shows that the distance between two cobalt atoms in the as-synthesized nanocrystals is similar to that in bulk cobalt metal (2.52 $\AA$ ). No cobalt atoms linking to oxygen atoms have been observed in the materials. However, due to the nature of the reducing agent, boron may still be present in the cobalt matrix. ${ }^{12}$ The size and its distribution are $7 \mathrm{~nm}$ and $17 \%$, respectively. The nanoparticles are dispersed in hexane, forming an optically transparent solution of concentration $5 \times 10^{-7} \mathrm{M}$. To selfassemble, the sample is prepared as follows: As a substrate, highly oriented pyrolytic graphite (HOPG) (for SQUID experiments) or a TEM grid covered by amorphous carbon (for characterization) is deposited in the bottom of a cell containing $200 \mu \mathrm{L}$ of $5 \times 10^{-7} \mathrm{M}$ cobalt nanocrystal solution. The solution evaporates slowly, yielding the formation of the monolayer. ${ }^{12-14,18}$

2.3. Annealing Process. At the end of the synthesis, the assynthesized cobalt nanocrystals coated with lauric acid are dispersed in hexane. The solvent of the colloidal solution is evaporated in a glovebox in nitrogen. The beaker with the remaining dark film made of uncoalesced cobalt nanocrystals is then annealed at $275^{\circ} \mathrm{C}$ for $15 \mathrm{~min}$ under nitrogen (Scheme 1). After fast-cooling at room temperature, hexane is added to the beaker, and the solution is subjected to sonication. A small fraction of the films remains in the bottom of the beaker, but most of the material is dispersed, forming a stable and optically clear solution. This method is limited to an annealing temperature up to $300{ }^{\circ} \mathrm{C}$ and an annealing time of no more than $30 \mathrm{~min}$. Above these values, it is impossible to disperse the nanocrystals again in hexane. The sample is introduced in the oven only when the desired temperature is reached to minimize the annealing time. Then, the sample is fast-cooled at room temperature in a glovebox.

2.4. Magnetic Measurements and Analysis. The magnetic properties of the nanocrystals either isolated in solution or selfassembled on a HOPG substrate are studied by SQUID. The susceptibility behavior is measured under zero-field-cooled (ZFC) and field-cooled (FC) conditions. The ZFC/FC experiment allows us to make a quantitative study on the annealing effect on supported and isolated nanocrystals. In the ZFC, the sample is cooled to $3 \mathrm{~K}$ without applying the field starting from a temperature at which all of the particles are in the superparamagnetic state. Afterward, a 75 Oe field is applied, and the magnetization is measured as a function of the increased temperature. In the case of an ideal system of perfectly monodispersive particles, the magnetization measured in the ZFC curve drops upon cooling from a maximum to zero in a few degrees. The temperature $T_{\mathrm{b}}$ at which the susceptibility peak occurs represents the particle blocking temperature that is related to the particle magnetic anisotropy energy (MAE) $K V$ by the relation $K V=k_{\mathrm{B}} T_{\mathrm{B}} \ln \left(1 / f \tau_{0}\right) \approx 28 k_{\mathrm{B}} T_{\mathrm{B}}$, where $V$ is the particle volume, $K$ is the magnetic anisotropy energy per volume unit, and $\tau_{0} \approx 10^{-9}-10^{-11} \mathrm{~s}^{19}$ The width of this transition (i.e., the distribution of MAE) becomes larger with increasing size dispersion of the particles. For nanocrystals having large size distribution, the volume $V$ has to be replaced by an effective volume $V_{\text {eff }}$ defined to be $V_{\text {eff }}=\left\langle V^{2}\right\rangle /\langle V\rangle .{ }^{20}$ This analysis gives an average value of the MAE.

The hysteresis curves are recorded at $3 \mathrm{~K}$ far away from the superparamagnetic state, and the field direction is kept parallel to the plane of the self-assembled layer.

\section{Results and Discussion}

3.1. Effect of Annealing on the Nanoparticle Structure. As described previously, ${ }^{13,14}$ cobalt nanocrystals with an average diameter of $7 \mathrm{~nm}$ and $17 \%$ size distribution synthesized via a colloidal solution (see Experimental Section) are coated with lauric acid, $\mathrm{CH}_{3}\left(\mathrm{CH}_{2}\right)_{10} \mathrm{COOH}$, and dispersed in hexane, forming an optically clear solution. The TEM image (Figures 1A, 2A, and 2B), obtained by deposition of a droplet of solution ([nanocrystals $]=5 \times 10^{-7} \mathrm{~mol} \mathrm{~L}^{-1}$ ), shows a $2 \mathrm{D}$ monolayer with a local ordering. The histogram (Figure 1C) confirms the average size of $7 \mathrm{~nm}$ and $17 \%$ size distribution. The corresponding selected area electron diffraction (SAED) pattern shows two diffuse rings at $\sim 2$ and $1.25 \AA$, indicating a low degree of crystallinity of the as-synthesized cobalt nanoparticles (Figure 1B). The high-resolution TEM (HRTEM) image recorded from the as-synthesized nanoparticle (Figure 3A) shows poor crystallinity, where the wavy lattice fringes are observed. It should be noticed that in some cases a shell is observed surrounding the nanocrystals (Figure 2A). This shell increases during long exposure to the electron beam in TEM. It is likely that the shell could come from the electron-beam-induced contamination, which may limit the possibility of HRTEM study on the as-synthesized nanocrystals. The shell cannot be due to an oxide layer because no ring at $2.46 \AA$ is observed in the SAED patterns. The observed shell, which presents a very low contrast in TEM, could also come from the lauric acid surrounding the nanocrystals. It forms a passivating layer because the carboxylic group is strongly attached to the surface cobalt atoms, and it blocks the oxidation process. As a matter of fact, without this passivating agent, the nanocrystals are oxidized in reverse micelles in $30 \mathrm{~min},{ }^{21}$ while it took at least 1 week for the lauric-acid-passivated nanocrystals. X-ray diffraction spectrum is obtained from a powder made of cobalt nanocrystals prior their redispersion in hexane (Figure 4, open circle). The diffractogram, obtained in open air, confirms the poor crystallinity of the as-synthesized cobalt nanoparticles, and no trace of oxide is observed. This result agrees with the EXAFS characterization performed on the as-synthesized nanocrystals, where no oxide was observed. ${ }^{13}$ However, as mentioned above, it cannot exclude the presence of boron in the matrix of the as-synthesized cobalt. ${ }^{12}$

We now describe the effect of the annealing on the structure of the nanoparticles. The nanocrystals are annealed at $275{ }^{\circ} \mathrm{C}$ in an oven for $15 \mathrm{~min}$ (under nitrogen) and then redispersed in 


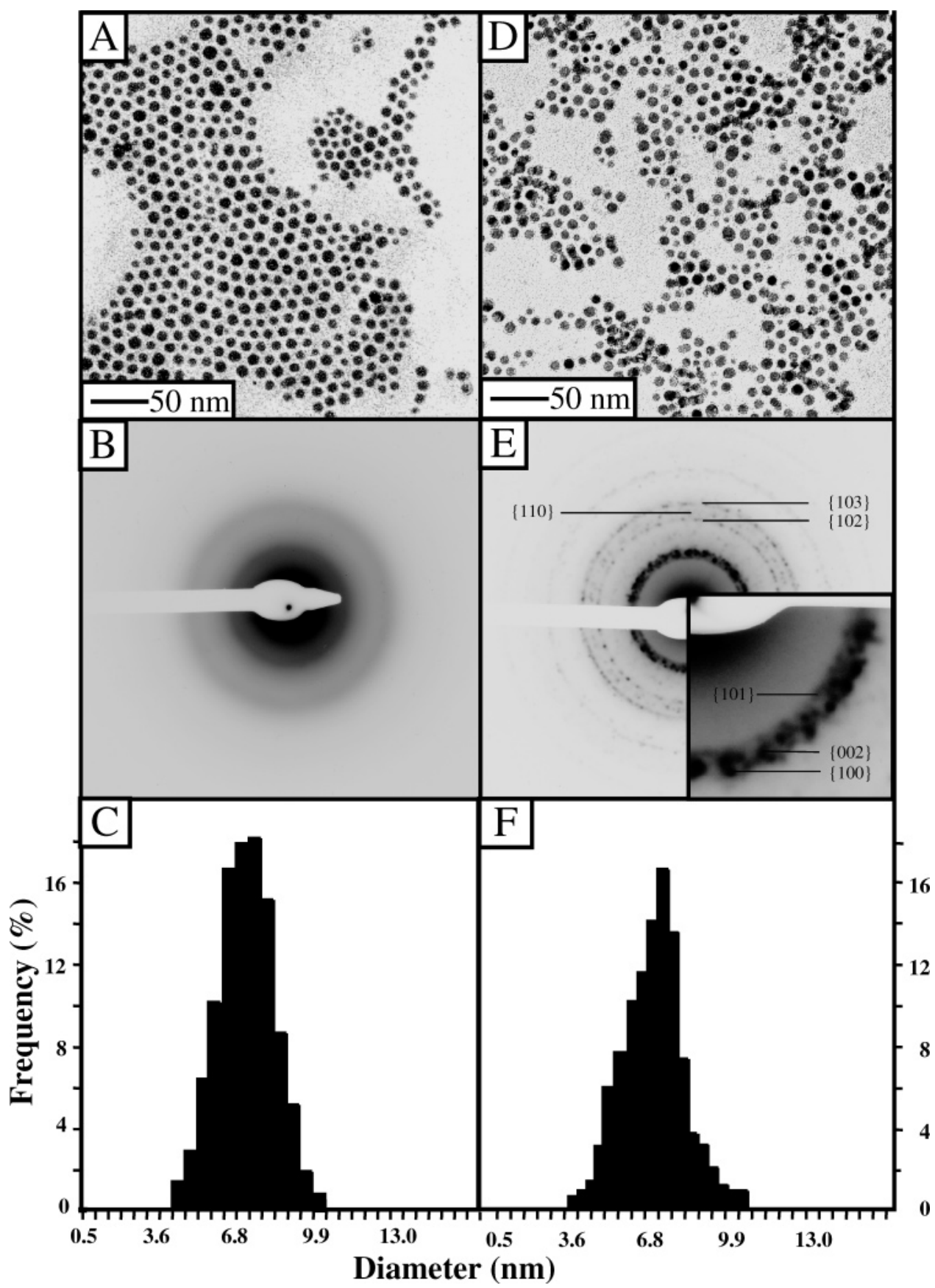

Figure 1. TEM images (A and D), SAED patterns (B and E), and size distributions (C and F) of the as-synthesized nanoparticles (A, B, and C) and nanocrystals annealed at $275{ }^{\circ} \mathrm{C}(\mathrm{D}, \mathrm{E}$, and $\mathrm{F})$. The inset of part $\mathrm{E}$ is a magnification of the SAED patterns showing the characteristic lines of the hcp cobalt nanocrystals.

hexane after fast-cooling (see above), forming a stable optical transparent solution. The TEM image (Figures 1D, 2C, and 2D) shows nanocrystals dispersed on the substrate. The average diameter and the size distribution (Figure 1F) decrease slightly to $6.8 \mathrm{~nm}$ and $15 \%$, respectively. The differences in size and distribution are explained by the fact that after annealing some nanocrystals remain in the bottom of the vessel and cannot be dispersed in hexane. This is due to desorption of some lauric acid molecules at the nanocrystal interface during annealing. This is possible because the boiling temperature of lauric acid is rather low $\left(225^{\circ} \mathrm{C}\right)$ compared to the annealing temperature. TEM images clearly indicate that no coalescence takes place after this fast and moderate annealing. The SAED (Figure 1E) shows spots forming a pattern of rings at 2.13, 2.02, 1.89, 1.46, 1.24, 1.14, and $1.05 \AA$ (Table 1). This corresponds to the diffraction pattern of the hep structured Co, ${ }^{22} 2.16,2.03,1.91$, $1.48,1.25,1.14$, and $1.04 \AA$ (Table 1). External rings due to graphitic carbon are discernible, and no additional ring at $2.46 \AA$ is observed, indicating the absence of cobalt oxide (Table 1 ). The X-ray powder diffraction pattern obtained on a powder of annealed cobalt nanoparticles prior their redispersion in hexane confirms the drastic evolution of the crystallographic structures (Figure 4, solid line). The sharp peaks after annealing characterize the much improved crystallinity of the nanoparticles, in agreement with the TEM observation shown in Figures 2 and 3. In comparison to electron diffraction, XRD characterizes a large number of particles, and due to the random orientation of the nanoparticles, XRD corresponds to an average diffraction pattern of the nanocrystals. It can be observed that the diffraction pattern obtained locally by SAED (Figure 1E) 


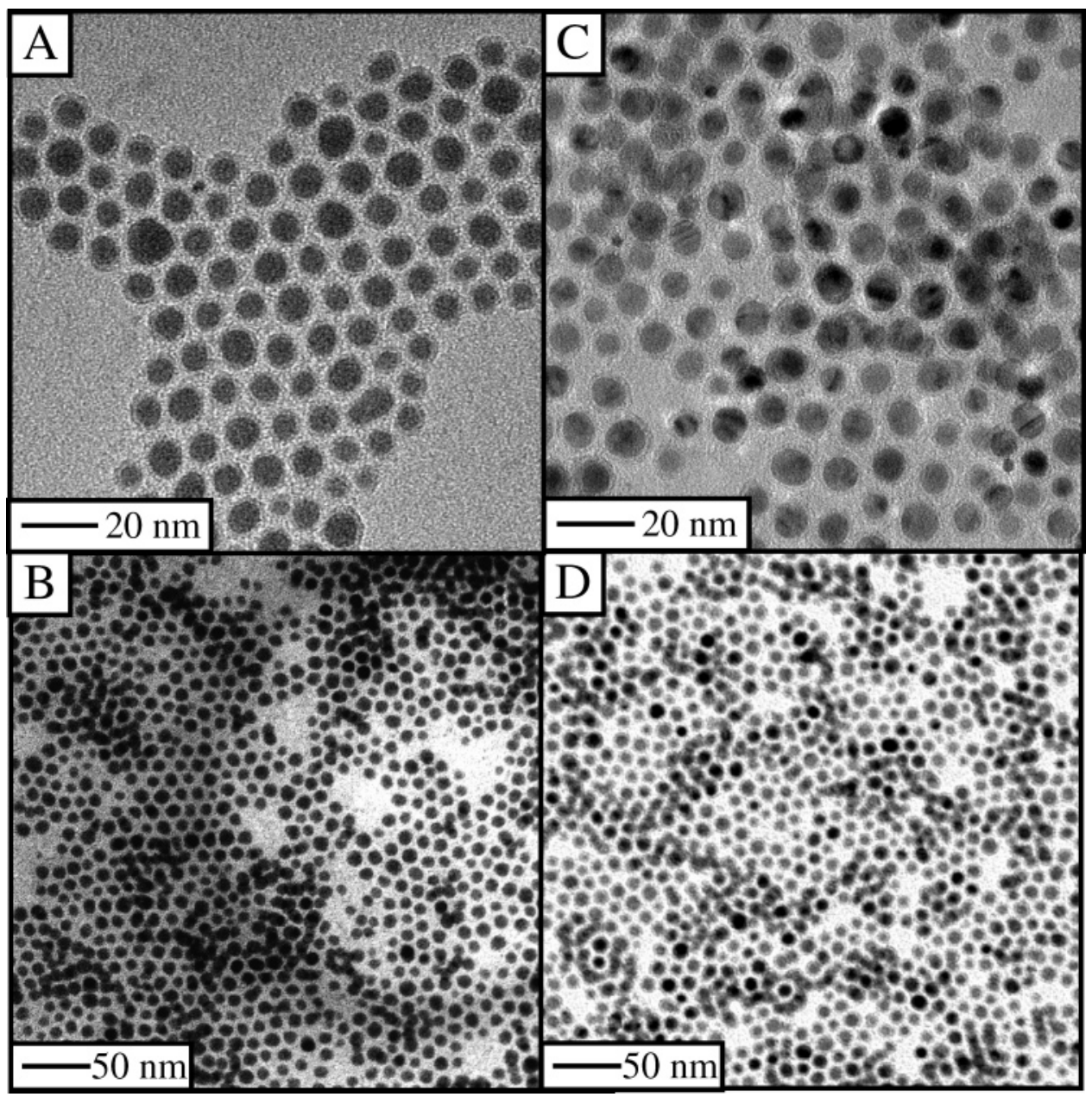

Figure 2. TEM images at high (A and C) and low magnification (B and D) of 2D monolayers made by as-synthesized cobalt nannocrystals (A and $\mathrm{B})$ and annealed nanocrystals (C and $\mathrm{D})$, respectively.

fits exactly the XRD pattern (Figure 4, dotted line). Thus, the two experiments give the same result at either the microscopic or the macroscopic level. The intensity and the position of the peaks exclude the formation of the $\epsilon$ phase $\mathrm{Co}^{23,24}$ as well as fcc cobalt nanocrystals. ${ }^{24}$ In fact, if a significant part of the nanocrystals are in the fcc form, then a peak at $1.77 \AA\left(60.6^{\circ}\right)$ should be observed, but this is not the case (Figure 4 and Table 1). These data are consistent with the reported data and simulation of cobalt in hcp forms. ${ }^{24}$ However, no peaks due to oxide can be observed in the region where there is no superposition of the different peaks $\left(42^{\circ}\right.$ or $\left.2.46 \AA\right)$. This confirms that the shell observed locally on the TEM grid is not due to an oxide shell but more likely due to the coating agent and the carbon substrate on the TEM grid. HRTEM allows refinement of the picture of the annealed nanocrystals. The nanocrystals show strong diffraction contrast, which is absent prior to annealing (Figure 2). Such strong diffraction effects clearly confirm the high crystallinity of the nanoparticles. The contrast suggests that some of the particles are composed of multiple grains. In fact, the HRTEM images (Figures 3B and 3C) show formation of monocrystals with an interlattice distance of $2 \pm 0.05 \AA$, consistent with the $2.02 \AA$ lattice spacing for the (002) planes and the $1.91 \AA$ spacing for the (101) planes of hcp cobalt. The particle in Figure $3 \mathrm{~B}$ is composed of grains that are linked by stacking faults and twins, as suggested by the bending of the lattice fringes across the particle. The particle in Figure $3 \mathrm{C}$ is likely to be single-crystalline.

It can be concluded that gentle annealing of the nanoparticles produces hcp structured nanocrystals with high crystallinity, which are easily redispersed in a solvent, while the average size and size distribution are preserved. An important fact is that the annealing process does not destroy the ability of nanocrystals to form 2D monolayers. Figure 2D shows the characteristic organization of annealed nanocrystals deposited on an amorphous carbon film on a TEM grid. The order of self-assembly is close but lower than that of the cobalt nanocrystals prior to annealing (Figure 2B). This is probably due to a partial degradation or desorption of the passivating agent during the annealing process. The lack of a passivating agent likely induces the lower degree of ordering of the annealed nanocrystals (Figure 2 ), because the passivation agent serves the interparticle linkage and "bonding". 18,25,26

3.2. Magnetic Properties of the Annealed Cobalt Nanocrystals Isolated and/or Self-Assembled in 2D. We now compare the magnetic properties of cobalt nanoparticles ([nanoparticles $\left.]=5 \times 10^{-7} \mathrm{M}\right)$, which are as-synthesized or annealed at $275^{\circ} \mathrm{C}$ and dispersed in hexane. The ZFC/FC curves clearly show a shift of the blocking temperature from 65 to $215 \mathrm{~K}$ on annealing (Figure 5A, Table 2). No peak at $8 \mathrm{~K}$ is observed for both the as-synthesized and the annealed nanocrystals, indicating the absence of cobalt oxide in the sample. ${ }^{23}$ The relative peak width of the ZFC/FC curve, (i.e., the distribution of MAE) decreases for annealed nanocrystals compared to that of the assynthesized material (insert Figure 5A). This is mainly due to the higher homogeneity of the crystalline structure of the nanocrystals and cannot be explained by a slight change in the size distribution (from $17 \%$ to $15 \%$ ) or in the average diameter (from 7 to $6.8 \mathrm{~nm}$ ). In fact, the presence of larger nanocrystals 


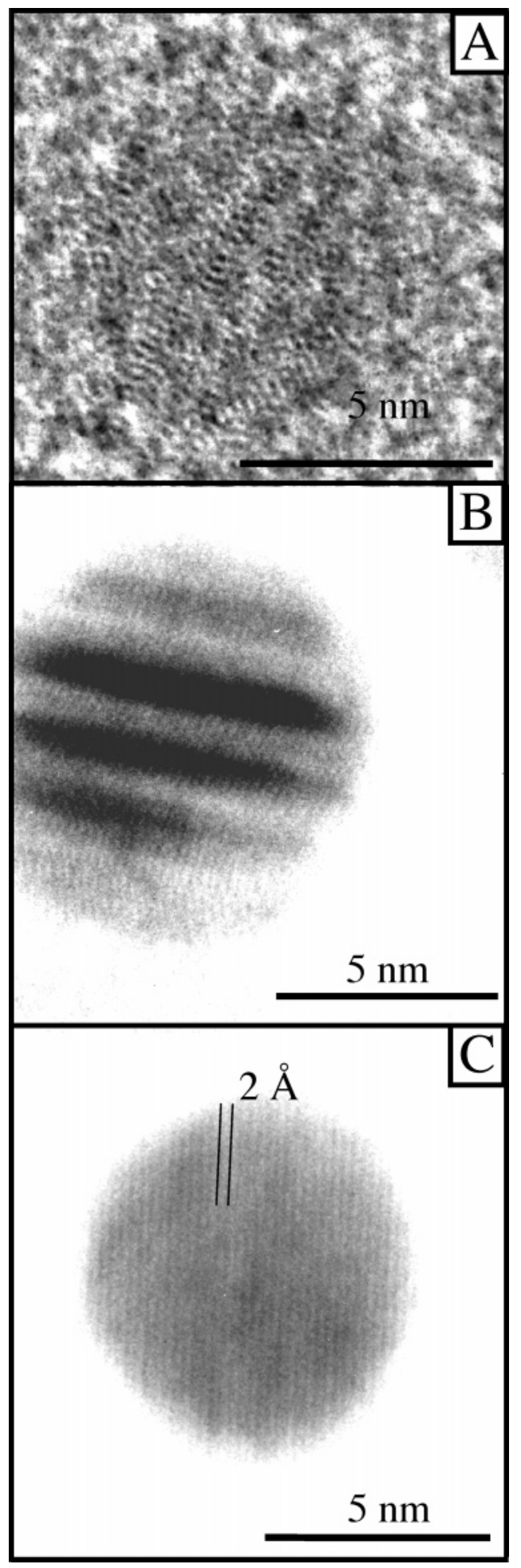

Figure 3. HRTEM images of nanocrystals (A), as-synthesized nanocrystals (B), and nanocrystals after annealing at $275^{\circ} \mathrm{C}(\mathrm{C})$.

as well as dimmers or sintered nanocrystals should increase the width of the MAE. The blocking temperature deduced from the $\mathrm{ZFC} / \mathrm{FC}$ studies is related to the magnetic anisotropy energy constant, $K_{\mathrm{a}}$, by the relation of $K_{\mathrm{a}} V \approx 28 k_{\mathrm{B}} T_{\mathrm{B}} \cdot{ }^{20}$ This constant is equal to $1.35 \times 10^{6}$ and $4.50 \times 10^{6} \mathrm{erg} / \mathrm{cm}^{3}$, respectively, for the samples before and after annealing at $275^{\circ} \mathrm{C}$. The first value is consistent with the previously reported value for cobalt nanocrystals with a low crystallinity ${ }^{11,27}$ or in the $\epsilon$ phase. ${ }^{7}$ The second value corresponds to the magnetic anisotropy of cobalt metal in the hcp structure $\left(4.7 \times 10^{6} \mathrm{erg} / \mathrm{cm}^{3}\right) .{ }^{28}$ It should be noted that in case of fcc cobalt this value should be $2.7 \times$ $10^{6} \mathrm{erg} / \mathrm{cm}^{3} .{ }^{29}$ The structure change is really a progressive phase transition as demonstrated by considering the magnetic properties of cobalt nanocrystals annealed at a lower temperature, $250{ }^{\circ} \mathrm{C}$. The $\mathrm{ZFC} / \mathrm{FC}$ curve shows two broad bands (Figure $5 \mathrm{~A}$, dotted line). The first one is centered at $85 \mathrm{~K}$ and corresponds to the as-synthesized nanocrystals, and the second one is centered a $190 \mathrm{~K}$ and corresponds to nanocrystals partially recrystalized in the hcp phase. Hence, a transition is really obtained from soft magnetic cobalt nanocrystals to hard magnetic cobalt nanocrystals.

The magnetization curve, at $3 \mathrm{~K}$, before and after annealing at $275{ }^{\circ} \mathrm{C}$ shows drastic changes (Figure 6A, Table 2). It should be noted that in both cases the hysteresis loops do not change if the hysteresis is obtained by cooling the sample under magnetic field (FC) or without magnetic field (ZFC). This confirms that there is no oxide shell coating on the nanocrystals. ${ }^{24}$ For the as-synthesized nanoparticles, the saturation magnetization, $M_{\mathrm{s}}$, not reached at $2.5 \mathrm{~T}$, is estimated from the $M$ versus $1 / H$ curve to be $85 \pm 5 \mathrm{emu} / \mathrm{g}$. Conversely, it is reached at $2 \mathrm{~T}$ for annealed nanocrystals and is markedly increased $(145 \pm 5 \mathrm{emu} / \mathrm{g})$ compared to the value for the assynthesized nanoparticles. The coercivity and reduced remanence are $0.18 \mathrm{~T}$ and 0.35 for the as-synthesized nanoparticles, while they are $0.13 \mathrm{~T}$ and 0.42 for the annealed materials. In the latter case, the reduced remanence is close to the theoretical value for nanocrystals having uniaxial anisotropy such as cobalt in the hcp form $\left(M_{\mathrm{r}} / M_{\mathrm{s}}=0.5\right)$, while it is 0.8 for the fcc structured Co. The major change between the as-synthesized and annealed nanocrystals is observed in the saturation magnetization value, which increases from 85 to $145 \mathrm{emu} / \mathrm{g}$. This is explained as follows: The as-synthesized nanoparticle is made of small crystalline domains separated by amorphous cobalt or cobalt/boron (Figure 3A). Each crystalline domain is characterized by its own magnetic moment. The total magnetic moment of the nanoparticle is the sum of these magnetic domains coupled by dipolar interactions. As a result, a low value of $M_{\mathrm{s}}$ is obtained. After the annealing, homogeneous nanocrystals are formed, and these nanodomains disappear (Figures 3B and 3C). A monocrystalline phase, characterized by a uniaxial moment, is produced. The saturation magnetization $(145 \mathrm{emu} / \mathrm{g})$ and reduced remanence $(0.43)$ are then close to those of the bulk phase (162 emu/g and 0.5). A slight difference between these two values is attributed to the surface effect and the adsorbed coating agent.

Due to the soft annealing process described above, the coating layer is preserved, and the magnetic properties of the isolated nanocrystals can be compared to those obtained when they are deposited on a substrate to form a self-organization. In previous papers, we demonstrated that, as a result of the dipolar interaction, the magnetic properties of an assembly of the assynthesized nanocrystals deposited on a substrate differ from those obtained with the same nanocrystals dispersed in a solvent (without interactions between nanocrystals). ${ }^{30,31}$ This is welldemonstrated below for both the as-synthesized and annealed nanocrystals (Table 2). In fact, with as-synthesized cobalt nanocrystals, the blocking temperature (Figure 5, solid line) and the reduced remanence (Figure 6, dotted line) increase from 65 to $80 \mathrm{~K}$ and from 0.35 to 0.49 , respectively, when the nanocrystals are isolated and deposited on HOPG (Table 2). This result agrees with the data published previously. ${ }^{12-14,30,31}$ For the $275^{\circ} \mathrm{C}$ annealed nanocrystals, the blocking temperature increases from 215 to $275 \mathrm{~K}$ when the nanocrystals are deposited on a substrate (Table 2). As expected, the hysteresis loop is 


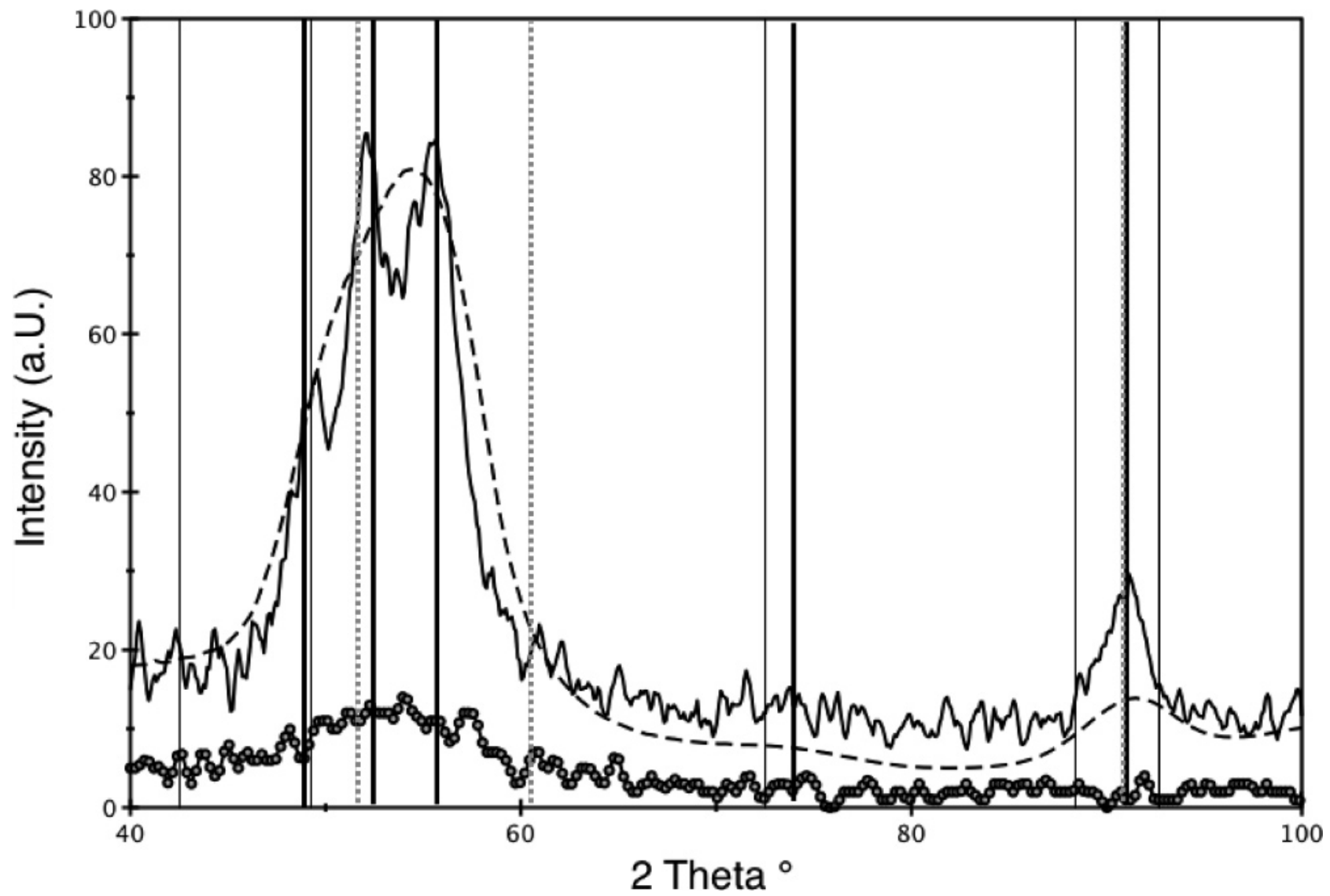

Figure 4. X-ray diffraction patterns from powders made of the as-synthesized cobalt nanocrystals (open circles) and nanocrystals after annealing at $275{ }^{\circ} \mathrm{C}$ (solid line). The dotted line corresponds to the SAED patterns shown in Figure 1E, which was radially averaged and converted in a 2-theta plot. The vertical line corresponds to the tabulated positions of the hcp cobalt (bold line), cobalt oxide (solid line), and fcc cobalt (doted line).

TABLE 1: SAED and XRD Characterization of the Annealed Cobalt Nanocrystals

\begin{tabular}{|c|c|c|c|c|c|c|c|c|c|c|}
\hline $\operatorname{SAED} d_{h k l}{ }^{a}$ & $\mathrm{XRD} d_{h k l}{ }^{a}$ & \multicolumn{3}{|c|}{$\mathrm{CO}_{\text {hcp }}$ line intensity spacing ${ }^{b}$} & \multicolumn{3}{|c|}{$\mathrm{Co}_{\mathrm{fcc}}$ line intensity spacing ${ }^{c}$} & \multicolumn{3}{|c|}{ CoO line intensity spacing ${ }^{d}$} \\
\hline & & & & & & & & 2.46 & $\{111\}$ & 75 \\
\hline 2.13 & 2.14 & 2.16 & $\{100\}$ & 27 & & & & 2.13 & $\{200\}$ & 100 \\
\hline 2.02 & 2.04 & 2.02 & $\{002\}$ & 30 & 2.05 & $\{111\}$ & 100 & & & \\
\hline 1.89 & 1.92 & 1.91 & $\{101\}$ & 100 & & & & & & \\
\hline 1.46 & & 1.48 & $\{102\}$ & 2 & & & & 1.51 & $\{220\}$ & 50 \\
\hline 1.25 & 1.25 & 1.25 & $\{110\}$ & 10 & 1.25 & $\{220\}$ & 25 & 1.28 & $\{311\}$ & 20 \\
\hline 1.14 & & 115 & $\{103\}$ & 10 & & & & 1.23 & $\{222\}$ & 15 \\
\hline 1.05 & & 1.04 & $\{201\}$ & 15 & 1.07 & $\{311\}$ & 30 & & & \\
\hline
\end{tabular}

${ }^{a} d_{h k l}$ in $\AA$ in comparison to the reference materials. ${ }^{b}$ Co hcp JCPDS collection code 5-727. ${ }^{c}$ Co fcc JCPDS collection code $15-806 .{ }^{d}$ CoO JCPDS collection code 9-402.

more square, and the reduced remanence increases from 0.45 to 0.52 with a slight decrease in the coercivity (from 0.13 to $0.11 \mathrm{~T})$. These changes in the magnetic properties are attributed to dipolar interaction among nanocrystals. ${ }^{31}$ The inset in Figure 5B superimposes the normalized ZFC curve (i.e., $M$ vs $T / T_{\mathrm{b}}$ ) for a $2 \mathrm{D}$ monolayer of the as-synthesized and annealed nanocrystals. This invariance of the MAE also excludes the presence of large nanocrystals due to coalescence. This behavior markedly differs from the ZFC curve of the same nanocrystals dispersed in solution with a decrease in the width of the ZFC curve. This is explained by the fact that the dipolar interactions do not markedly differ after annealing. This is estimated by taking into account the coupling dipolar interaction constant, defined as the ratio of the dipolar to the anisotropy energies. Since the saturation magnetization markedly increases by annealing, we would expect, from the coupling constant $\alpha_{\mathrm{d}}=$ $\left(\pi M_{\mathrm{s}}^{2} / 12 K_{\mathrm{a}}\right)(D / d)^{3}$ (deduced from Stoner-Wolfarth particles where $D$ and $d$ are the average diameter and the interparticle distance, $M_{\mathrm{s}}$ in $\mathrm{emu} / \mathrm{cm}^{3}$ (i.e. emu/g $\times$ density), respectively, to observe a change in the magnetization loop. ${ }^{31}$ However, the increase in the crystallinity of nanocrystals produced by annealing induces an increase in the anisotropy constant, $K_{\mathrm{a}}$, by a factor of 3.2. This compensates for the increase in the saturation magnetization, and the coupling constant remains quite unchanged. It is 0.04 and 0.036 for as-synthesized and annealed nanocrystals.

The dipolar interactions between adjacent nanocrystals also explain the invariant of the width of the ZFC curve when nanocrystals are deposited in 2D monolayers, as the long-range scale dipolar interactions average the individual behavior. As demonstrated previously, ${ }^{31}$ the local ordering in the monolayer does not play a visible role in the collective magnetic properties. Hence, the loss of organization observed for the annealed nanocrystals does not modify the interaction. The ZFC/FC curve 


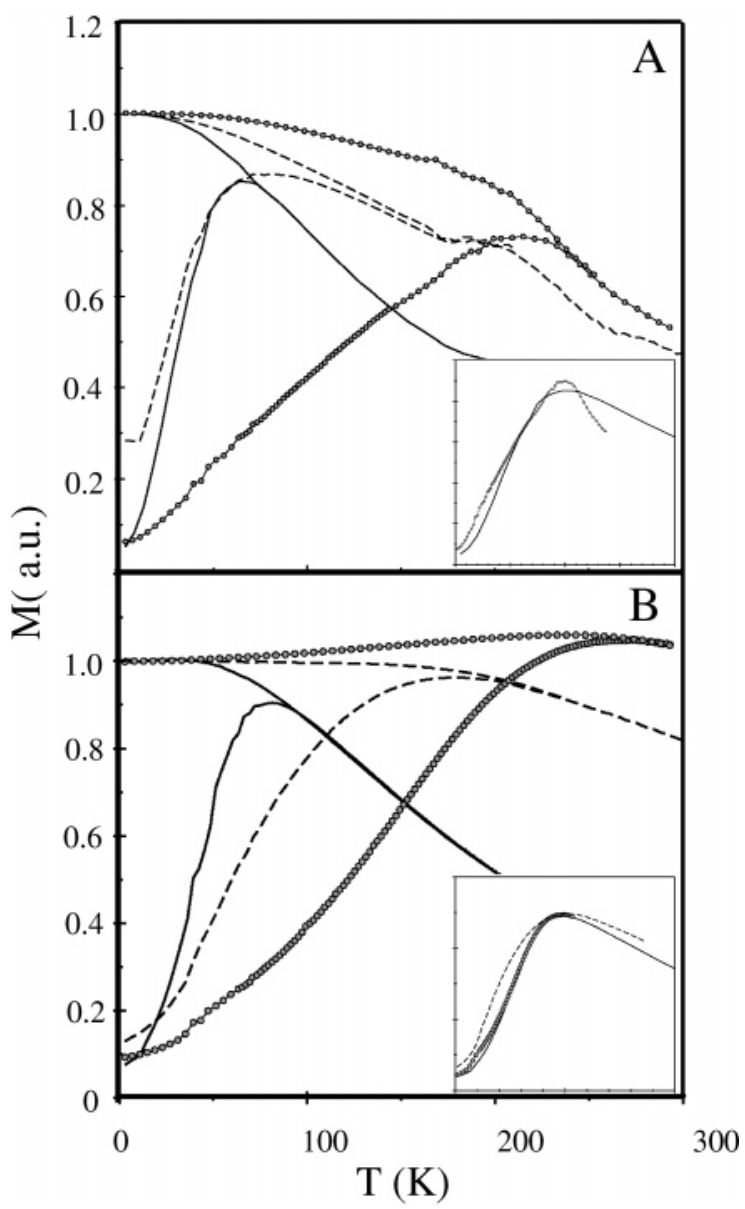

Figure 5. $\mathrm{ZFC} / \mathrm{FC}$ curves of the as-synthesized (solid line), $250{ }^{\circ} \mathrm{C}$ annealed (dotted line), and $275{ }^{\circ} \mathrm{C}$ annealed (open circles) cobalt nanocrystals The inset corresponds to the normalized MAE curves ( $M$ vs $T / T_{\mathrm{b}}$ ): (A) dispersed in hexane; (B) deposited on a HOPG substrate

TABLE 2: Magnetic Properties of Cobalt Nanocrystals after Different Treatment and Processing

\begin{tabular}{lcrcc}
\hline \multicolumn{1}{c}{ sample } & $T_{\mathrm{b}}(\mathrm{K})$ & $M_{\mathrm{s}}(\mathrm{emu} / \mathrm{g})$ & $H_{\mathrm{c}}(\mathrm{T})$ & $M_{\mathrm{r}} / M_{\mathrm{s}}$ \\
\hline as-synthesized Co isolated & 65 & $85 \pm 5$ & 0.18 & 0.35 \\
Co as-synthesized in 2D monolayers & 80 & $85 \pm 5$ & 0.18 & 0.49 \\
Co hcp isolated (annealed at $\left.275^{\circ} \mathrm{C}\right)$ & 215 & $145 \pm 5$ & 0.13 & 0.45 \\
Co hcp in 2D monolayer & 275 & $145 \pm 5$ & 0.11 & 0.52
\end{tabular}

of the nanocrystals annealed at $250^{\circ} \mathrm{C}$ and deposited on HOPG shows a broad single band centered at $175 \mathrm{~K}$ but not the two peaks as observed when the nanoparticles are dispersed in solution (see above). This is explained in terms of long-range dipolar interactions. The interactions are averaged over the substrate regardless the state of crystallinity of the particles. As a result, the two bands of the two states of the nanocrystals fuse into a broader band. This is confirmed by the increase in the MAE width compared with those of the as-synthesized nanocrystals and those annealed at $275^{\circ} \mathrm{C}$ (inset in Figure 5B).

Hence, the drastic change in the magnetic properties of the individual nanocrystals is obtained without changing the dipolar interaction. This is an important point for future devices based on the organization of hcp cobalt nanocrystals.

\section{Conclusion}

By a novel annealing process, the partially crystallized 7-nm cobalt nanoparticles have been completely transformed into 7-nm hexagonal nanocrystals that preserve the size, size distribution, and ability to form 2D self-organization without

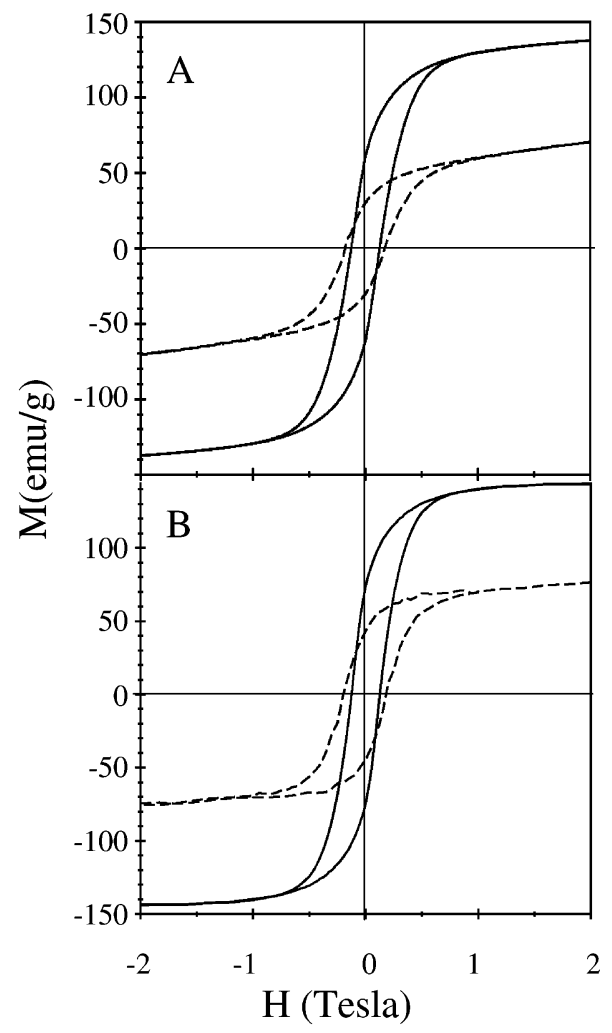

Figure 6. Comparison of the hysteresis loops at $3 \mathrm{~K}$ from the assynthesized (dotted line) and $275{ }^{\circ} \mathrm{C}$ annealed (solid lines) cobalt nanocrystals: (A) isolated nanoparticles in hexane solution $(5 \times$ $10^{-7} \mathrm{~mol} \mathrm{~L}^{-1}$ ); (B) nanoparticles deposited on a HOPG substrate.

agglomeration or coalescing. The postannealed nanocrystals have a high magnetic anisotropy and high crystallinity in a pure hcp structure. This is a key progress in preparation of magnetic nanoparticles that have practical important magnetic properties. Because these nanocrystals are dispersed in a solvent, it is possible to fully characterize the structural and magnetic changes induced by the soft annealing. Moreover, as the coating molecules are preserved, the deposition on a substrate is quite easy. Collective magnetic properties of the monolayers made of hcp cobalt nanocrystals indicate that the strength of the dipolar interactions is unchanged by the annealing. This is an important point for future applications. The blocking temperature of the monolayer is now $275 \mathrm{~K}$, close to that required for technological applications $(300 \mathrm{~K})$. This work could open many applications for using small size ferromagnetic nanocrystals.

\section{References and Notes}

(1) Special Issue on Nanoscale Materials. Acc. Chem. Res. 1999, 32, $387-454$.

(2) Lambeth, D. N.; Velu, E. M. T.; Bellesis, G. H.; Lee, L. L.; Laughlin, D. E. J. Appl. Phys. 1996, 79, 4496.

(3) Schwalom, D. D.; von Molnar, S. Physical Properties of NanometerScale Magnets. In Nanotechnology; Timp, G. L., Ed.; Springer: New York, 1999; Chapter 12, pp 437-470.

(4) Sun, S.; Murray, C. B.; Weller, D.; Folks, L.; Moser A. Science 2000, 287, 1989.

(5) Stahl, B.; Gajbhiye, N. S.; Wilde, G.; Kramer, G.; Ellrich, J.; Ghafari, M.; Hahn, H.; Gleiter, H.; Wei $\beta$ müller, J.; Würschum, R.; Schlossmacher, P. Adv. Mater. 2002, 14, 24.

(6) Chen, M.; Nikles, D. E. J. Appl. Phys. 2002, 91, 8477.

(7) Murray, C. B.; Sun, S.; Gaschler, W.; Doyle, H.; Betley, T. A.; Kagan, C. R. IBM J. Res. Dev. 2001, 45, 47 1788.

(8) Dinega, D. P.; Bawendi, M. G. Angew. Chem., Int. Ed. 1999, 38,

(9) Park, J.; Kang, N.-J.; Jun, Y.-W; Oh, S. J.; Ri, H.-C.; Cheon, J. ChemPhysChem 2002, 6, 543 
(10) Puntes, V. F.; Gorostiza, P.; Aruguete, D. M.; Bastus, N. G.; Alivisatos, A. P. Nat. Mater. 2004, 3, 263

(11) Margeat, O.; Amiens, C.; Chaudret, B.; Lecante, P.; Benfield, R. E. Chem. Mater. 2005, 17, 107.

(12) Petit, C.; Taleb, A.; Pileni, M. P. J. Phys. Chem. B 1999, 103, 1805.

(13) Legrand, J.; Petit, C.; Bazin, D.; Pileni, M. P. Appl. Surf. Sci. 2000, 164,186 ,

(14) Legrand, J.; Petit, C.; Pileni, M. P. J. Phys. Chem. B 2001, 105 5643.

(15) Wang, Z. L.; Dai, Z. R.; Sun, S. Adv. Mater. 2000, 12, 19441946.

(16) Glavee, G. N.; Klabunde, K. J.; Sorensen, C. M.; Hadjipanayis, G. C. Inorg. Chem. 1993, 32, 474

(17) Gangopadhyay, S.; Hadjipanayis, G. C.; Sorensen, C. M.; Klabunde, K. J. J. Appl. Phys. 1993, 73, 6964.

(18) Motte, L.; Billoudet, F.; Pileni, M. P. J. Phys. Chem. 1995, 99, 16425 .

(19) Tronc, E. Nuovo Cimento Soc. Ital. Fis., D 1996, 18, 163.

(20) Dormann, J. L.; D’Orazio, F.; Lucari, F.; Tronc, E.; Prené, P.; Lolivet, J. P.; Fiorani, D.; Cherkaoui, R.; Noguès, M. Phys. Rev. B 1996 $53,14291$.
(21) Petit, C.; Pileni, M. P. J. Magn. Magn. Mater. 1997, 166, 82.

(22) International Center for Diffraction File; Data File No. 050727; Joint Committee on Powder Diffraction Standards (JCPDS): Newton Square, PA.

(23) Bao, Y.; Beerman, M.; Pakhomov, A. B.; Krishnan, K. M. J. Phys. Chem. B 2005, 109, 7220.

(24) Puntes, V. F.; Zanchet, D.; Erdammez, C. K.; Alivisatos, A. P. J. Am. Chem. Soc. 2002, 142, 12874

(25) Wang, Z. L. Adv. Mater. 1998, 10, 13-30.

(26) Wang, Z. L.; Harfenist, S. A.; Whetten, R. L.; Bentley, J.; Evans, N. D. J. Phys. Chem. B 1998, 102, 3068-3072.

(27) Petit, C.; Rusponi, S.; Brune, H. J. Appl. Phys. 2004, 95, 4251

(28) Luis, F.; Torres, J. M.; Garcia, L. M.; Bartolomé, J.; Stankiewicz, J.; Petroff, F.; Fettar, F.; Maurice, J. L.; Vaurés, A. Phys. Rev. B 2002, 65, 094409.

(29) Chen, J. M.; Sorensen, C. M.; Klabunde, J. K.; Hadjipanayis, G. C. Phys. Rev. B 1995, 51, 11527

(30) Petit, C.; Taleb, A.; Pileni, M. P. Adv. Mater. 1998, 10, 259.

(31) Russier, V.; Petit, C.; Legrand, J.; Pileni, M. P. Phys. Rev. B. 2000, 62,3910 . 